ВІСНИК

ОДЕСЬКОГО НАЦІОНАЛЬНОГО

МОРСЬКОГО УНІВЕРСИТЕТУ
HERALD

OF THE ODESSA NATIONAL

MARITIME UNIVERSITY

№ $1(61), 2020$

УДК 621.431.74

DOI 10.47049/2226-1893-2020-1-131-139

\title{
ОПТИМАЛЬНОЕ УПРАВЛЕНИЕ СУДОВЫМ ДИЗЕЛЕМ ПРИ НЕСТАЦИОНАРНЫХ РЕЖИМАХ
}

\author{
A.В. Усов \\ д.т.н., профессор, заведующий кафедрой \\ Высшей математики и моделирования систем \\ Одесский национальный политехнический университет, Одесса, Украина

\section{Э.М. Половинка} \\ д.т.н., профессор, заведующий кафедрой \\ Судовые энергетические установки \\ Н.В. Слободянюк \\ преподаватель кафедры Корабельные электроэнергетические системы \\ Института Военно-Морских Сил \\ Одесская наџиональная морская академия, Одесса, Украина
}

Аннотация. На основании исследования управления топливной системы судовых дизелей при нестационарных режимах решена задача оптимизации расхода топлива в условиях динамики судового комплекса и состояния водной поверхности. Задача решена с использованием модели поведения судового двигателя при нестационарных режимах, содержащей основные технические характеристики двигателя, привода, нестационарности водной среды, оптимальным управлением топливной системы, обеспечивающим минимальный расход топлива при заданной средней скорости.

Ключевые слова: судовой комплекс, топливная система, модель, оптимальное управление.

\section{УДК 621.431.74}

DOI 10.47049/2226-1893-2020-1-131-139

\section{ОПТИМАЛЬНЕ УПРАВЛІННЯ СУДНОВИМ ДИЗЕЛЕМ ПРИ НЕСТАЦІОНАРНИХ РЕЖИМАХ}

\section{A.В. Усов}

д.т.н., професор, завідувач кафедри

Вищої математики та моделювання систем

Одеський національний політехничний університет, Одеса, Украӥна

\section{Е.М. Половинка}

д.т.н., професор, завідувач кафедри суднових енергетичних установок

\section{М.В. Слободянюк}

викладач кафедри Корабельної енергетики

та електроенергетичних систем Інституту Військово-Морських Сил

Одеська національна морська академія, Одеса, Україна

(С) Усов А.В., Половинка Э.М., Слободянюк Н.В., 2020 
Анотація. На основі дослідження управління топливної системи суднових дизелів на нестаціонарних режимах вирімена задача оптимізації витрат палива в умовах динаміки суднового комплексу та стану водневої поверхні. Задача розвязувалась з використанням моделі поведінки суднового двигуна на нестаиіонарних режсимах, в якій відображені тех-нічні характеристики двигуна,приводу, нестаиіонарності водного середовища оптимальним керуванням паливної системи, яке забезпечує мінімальні витрати топлива на середній швидкості.

Ключові слова: судновий комплекс, паливна система, модель, оптимальне управління.

UDC 621.431.74

DOI 10.47049/2226-1893-2020-1-131-139

OPTIMAL MANAGEMENT BY SHIP DIESEL AT NON-STATIONARY MODES

\author{
A. Usov \\ d.t.n., professor, managing the department of \\ higher mathematics and design of the systems \\ National polytechnic university, Odessa, Ukraine \\ E. Polovinka \\ D.t.n. professor of department . \\ N. Slobodiynyk \\ senior teacher of department
}

National University «Odessa Maritime Academy» Odesa, Ukraine

Abstract. On the basis of research of management of the fuel system of ship diesels at the non-stationary modes the task of optimization of expense of fuel is decided in the conditions of dynamics of ship complex and state of water surface. A task is decided with the use of model of behavior of ship engine at the non-stationary modes, containing basic technical descriptions of engine, drive, unstationarity of water environment, by the optimal management of the fuel system, providing the minimum expense of fuel at the set middle speed.

Keywords: ship complex, fuel system, model, optimal at rule.

Введение. Исследование основных динамических характеристик многих машин и механизмов можно проводить моделируя их как системы, имеющие две степени свободы. Это относится к таким энергетическим комплексам, как, например главные двигатели-гребные винты-корпус судна.

Вопросы совершенствования дизельных энергетических установок являются важнейшим трендом в протекании рабочего процесса, кото- 
рый включает в себя две составляющие: работа на установившихся режимах и работа на переменных режимах $[1 ; 2 ; 3]$.

Для энергетических установок, работающих в основном со стабильными нагрузками переменные режимы имеют большое значение, так как их характеристики определяют надежность и экономичность силовых установок в условиях маневрирования [4; $5 ; 6$ ]. Значительная часть времени эксплуатации двигателей внутреннего сгорания происходит в условиях изменяющихся режимов вследствие воздействия переменных нагрузок природного происхождения, таких как движение судна по большой волне или в ледовых условиях. Другим источником являются изменения, вносимые персоналом в эксплуатационный режим работы главного двигателя при маневрировании судна при входе в порт, прохождение каналов, движение с тралом или при буксировке.

Интенсивные изменения режимов работы главного двигателя оказывает существенное отрицательное влияние на протекающие рабочие процессы в циллиндрах, вспомогательных механизмах и агрегатах, а также на дополнительном расходе топлива.

Анализ последних исследований и публикаций. Одним из основных факторов, определяющих ухудшение эксплуатационных характеристик двигателей на переменных режимах, является изменение объема впрыскиваемого топлива [7; 8]. Это наиболее характерно в условиях переменной нагрузки на силовую установку.

Как указывалось в работах $[9 ; 10]$ переменные режимы являются неблагоприятными условиями работы двигателей.

Нестабильность работы топливной системы приводит к неодинаковой дозировке порции топлива по циллиндрам, перегрузке одних и недогрузке других циллиндров. А неодинаковые углы начала впрыскивания топлива по циллиндрам обуславливают разные значения максимального давления сгорания и температуры выпускных газов на эксплуатационных режимах.

На пусковых режимах при малых частотах вращения коленчатого вала и большой цикловой подачи в топливной системе высокого давления происходят сложные гидродинамические явления, вызывающие неустойчивую работу форсунок $[11 ; 12]$.

Целью настоящей работы является исследование оптимального управления топливной системой и разработка рекомендаций по оценке влияния топливной системы на рабочие и эксплуатационные характеристики судовых дизелей.

Изложение основного материала. Поведение главного двигателя в судовом комплексе при нестационарных режимах описывается следующей моделью, содержащей дифференциальные уравнения [13]:

$$
\frac{d v}{d t}=\frac{1}{A}[p(\omega, v, \varphi, t)-R(v)]
$$




$$
\frac{d v}{d t}=\frac{N}{A}\left[M_{g}(\omega, v)-M_{c}(\omega, v, \varphi, t)\right]
$$

с начальными условиями

$$
v(o)=1, \omega(o)=1 \text {. }
$$

В уравнениях (1)-(3), $v, t, p, \omega, M_{\mathrm{g}}, M_{\mathrm{c}}$ - относительные величины; $v$ - скорость движения судна; $t$ - время.

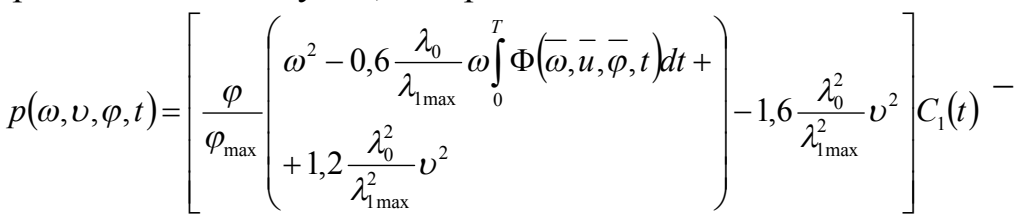

$$
\begin{aligned}
& M_{g}(\omega, u)=M_{c}\left(1,1, \varphi_{0}, o\right) M_{g}(\omega) u(t)-\text { движущий момент; } \\
& M_{c}(\omega, v, \varphi, t)=\left[\frac{\varphi}{\varphi_{\max }}\left(\omega^{2}-0,65 \frac{\lambda_{0}}{\lambda_{2 \max }} \omega v+1,45 \frac{\lambda_{0}^{2}}{\lambda_{2 \max }^{2}} v^{2}\right)-1,8 \frac{\lambda_{0}^{2}}{\lambda_{2 \max }^{2}} v^{2}\right] .
\end{aligned}
$$

$\frac{\lambda_{0}}{\lambda_{2 \max }}, A, N, \frac{\lambda_{0}}{\varphi_{\max }}-$ экспериментальные безразмерные коэффициенты;

$\varphi(t)$ - шаговое отношение винта;

$u(t)$ - отношение подачи топлива;

$C_{1}(t), C_{2}(t)$ - периодические функции, характеризующие нестандартность водной среды.

Необходимо найти управление $0 \leq u(t) \leq 1$, которое при установившихся режимах двигателя, обращало бы в минимум расход топлива на милю пути. Для этого требуется выполнение критерия

$$
\frac{1}{T} \int_{0}^{T}\left\{d+\left(M_{g} \omega-e\right)^{2}\left[\mu+v \sin n\left(M_{g} \omega-e\right)\right]\right\} M_{g} \omega d t \rightarrow \min
$$

при заданной средней скорости движения судна

$$
\frac{1}{T} \int_{0}^{T} v(t) d t=v_{1}
$$

Здесь $d, e, \mu, v$ определяются экспериментально при стендовых испытаниях двигателя.

Для решения задачи воспользуемся методом динамического программирования Беллмана [14]. Непосредственное применение метода Беллмана к задаче (1)-(3) вызывает большие вычислительные трудности, т.к. необходимо брать $T>>1$. Так как $1 / A$ мало по смыслу задачи, проведем усреднение системы. 
Уравнение первого приближения для $\bar{v}$ и соответствующее уравнение для $\omega$ при установившемся режиме двигателя (водная среда характеризуется стационарными колебаниями) имеют вид

$$
\begin{aligned}
& \int_{0}^{z n}\left[p\left(\bar{\omega}, v_{1}, \bar{\varphi}, t\right)-R\left(v_{1}\right)\right] d(t)=0 \\
& \frac{d \bar{\omega}}{d t}=\frac{N}{A}\left[M_{g}(\bar{\omega}, \bar{u})-M_{c}\left(\bar{\omega}, u_{1} \bar{\varphi}, t\right)\right] \\
& \omega(o)=\omega(2 \pi) .
\end{aligned}
$$

Введем множитель Лагранжа $\lambda$. Окончательно получим задачу о минимизации функционала.

$$
[u]=\int_{0}^{2 \pi} \Phi(\bar{\omega}, \bar{u}, \bar{\varphi}, t) d t \rightarrow \min .
$$

Здесь

$\Phi(\bar{\omega}, \bar{u}, \bar{\varphi}, t)=\left\{d+\left(M_{g} \omega-e\right)^{2}\left[\mu+v \sin n\left(M_{g} \bar{\omega}-e\right)\right]\right\} M_{g} \omega+\lambda(p-R)$ при условии (7)-(8). Значения $\lambda$ определяется из (6).

Сформулируем принцип оптимальности следующим образом. Пусть $M \epsilon K$, где $K$ - область определения функций управления.

Оптимальное управление обладает тем свойством, что каково бы ни было управление на множестве $M$, управление на множестве $K / M$ должно быть оптимальным относительно состояний, являющихся результатом применения управления на множестве $M$.

Введем функцию

$$
\begin{gathered}
F(\xi, \eta, T)=\int_{0}^{T} \Phi(\bar{\omega}, \bar{u}, \bar{\varphi}, t) d t+\int_{2 \pi-T}^{2 \pi} \Phi(\bar{\omega}, \bar{u}, \bar{\varphi}, t) d t \underset{u \in v}{\longrightarrow} \min \\
\bar{\omega}(T)=\xi, \quad \bar{\omega}(2 \pi-T)=\eta
\end{gathered}
$$

при выполнении (7)-(8).

Оптимальное управление по объему топлива на милю, в случае $N / A<<1$, соответствующее первому приближению для судовых систем имеет вид

$$
F(t)=a+8 \frac{C_{1}(t)}{C_{2}(t)}
$$

где $C_{1}(t), C_{2}(t)$ - возмущающие функции, при которых система автоматического регулирования поддерживает режим, близкий к оптимальному при действии произвольных возмущающих сил на корпус судна.

По принципу оптимальности имеем

$$
F(\xi, \eta, T+\Delta t)=\left\{\begin{array}{l}
\Delta t \\
\int \\
0 \\
+F[y(\Delta t) z(\Delta t, T)]
\end{array}\right\} \underset{u, v \in U}{\longrightarrow} \min
$$




$$
\begin{gathered}
F(\xi, \eta, \Delta t)=\int_{0}^{\Delta t^{\prime}}[\Phi(y, u, \varphi, \tau)+\Phi(z, v, \varphi, \tau)] d t \underset{u, v e U}{y(\Delta t)=\mathrm{z}(\Delta t)} \min \\
\frac{d y}{d \tau}=\frac{N}{A}\left[M_{g}(y, u)-M_{c}\left(y, v_{1}, \varphi, T+\Delta t-\tau\right)\right] \\
\frac{d z}{d \tau}=\frac{N}{A}\left[M_{g}(z, v)-M_{c}\left(z, v_{1}, \varphi, 2 \pi-T-\Delta t+\tau\right)\right] \\
y(o)=\xi \mathrm{z}(o)=\eta .
\end{gathered}
$$

Для решения (12)-(15) был использован метод последовательных приближений динамического программирования [15].

Проведенные исследования влияния параметра $N / A$ для судовых систем показали, что при увеличении значения N/A (увеличение подачи топлива в камеру сгорания) экспериментальные величины всех характеристик силовой системы асимптотически приближаются к некоторым $\approx \approx^{o} \approx \approx^{o}$

приделам. Так, при $N / A>5$ величины $M_{g}, M_{c}, P$ практически не зависят от $N / A$. Поэтому, для произвольного N/A можно задавать $F_{\min }$ и $F_{\max }$, полученные в предположении $N / A<<1$.

При $\Delta C_{2}>0,5$, т.е., для больших значений амплитуды возмущающей силы (амплитуда волновых колебаний водной среды), на промежутке $[0,1 ; 1,0]$ значения $F_{\max }$ и $F_{\min }$ достигают некоторых экстремальных величин, а затем монотонно уменьшаются. Если задавать в качестве $u_{1}$ значение скорости $u$, при $N / A>>1$ и $\Phi=\Phi_{0}$, то значение $\Phi_{\min }$ практически не зависит от $N / A$ при произвольном реальном значении $\Delta C_{2}$.

При полученных оптимальных управлениях величина снижения относительного расхода топлива достигает $5 \%$ по сравнению с соответствующим постоянным управлением. Анализ полученных результатов позволяет провести исследование влияния на динамику основных параметров, характеризующих элементы судового комплекса и состояние водной поверхности при оптимальном и постоянном управлении.

Экспериментальное подтверждение результатов моделирования управлением топливной системой силовой установки осуществлялось на дизелях судов-перегружателей речного флота. Так для главного судового дизеля с понижающим редуктором и пневматической системой дистанционного управления, предназначенный для установки на буксирах-кантовщиках и транспортно-крановых судах для работы на винт регулируемого шага при мощности 560 кВт, частое вращения 500/300 об/мин, удельном расходе топлива 211 г/кВтч при оптимальном и постоянном управлении можно достичь снижения расхода топлива до 200-192 г/кВтч, что составляет $5 \%$-6 \% по сравнению с соответствующим постоянным управлением. 
ВІСНИК

ОДЕСЬКОГО НАЦІОНАЛЬНОГО

МОРСЬКОГО УНІВЕРСИТЕТУ № 1 (61), 2020
HERALD

OF THE ODESSA NATIONAL

MARITIME UNIVERSITY № 1 (61), 2020

Введение управления по шагу винта приводит к дополнительному уменьшению расхода топлива 5 \%-6 \% при заданной скорости движения по сравнению с движением судна с наилучшим постоянным шагом винта или увеличению скорости судна до $10 \%$.

Выводы. Исследование оптимального управления топливной системой по оценке влияния ее на рабочие и эксплуатационные характеристики судовых дизелей позволило установить следующее.

1. При полученных оптимальных управлениях величина снижения относительного расхода топлива достигает до 5 \% по сравнению с соответствующим постоянным управлением.

2. Решение поставленной задачи дает возможность исследовать влияние основных динамических характеристик элементов судового комплекса в зависимости от состояния водной поверхности при оптимальном и постоянном управлении.

\section{СПИСОК ЛІТЕРАТУРИ}

1. Лепешкин Д.И. Экспериментальные исследования по осциллографированию прочессов в топливной аппаратуре дизеля / Д.И. Лепешкин, А.Л. Иванов // Омский научный вестник. № 3 (123). 2013. C.150-153.

2. Смирнов А.М. Метод диагностирования топливной аппаратуры высокого давления дизелей / А.М. Смирнов, С.В. Рослов, Е.С. Терещенко, Д.В. Щабалин // Омский научный вестник. № 3 (133). 2014. C.133-136.

3. Саенко М.М. Влияние различных факторов на величину и равномерность подачи топлива в циилиндры дизеля / М.М. Саенко, А.П. Жигадло, А.Л. Иванов // Вестник СибАДИ. № 4 (32). 2013. C.29-35.

4. Саенко М.М. Влияние конструкции приборов топливной системь дизеля на величину топливоподачи / М.М. Саенко // Вестник СибАДИ. № 6 (40). 2014. С.49-55.

5. Саенко М.М. Анализ существующих методов испьтаний приборов топливных систем дизелей при техническом обслуживании в процессе эксплуатациии / М.М. Саенко // Вестник СибАДИ. № 2 (42). 2015. С.40-46.

6. Потомарев М.Н. Переходные проиессы в линиях высокого давления топливной аппаратуры дизеля / М.Н. Пономарев, А.К. Синицын, Д.Е. Соловьев // Вестник РУДЫ, сер. Инженерные исследования. 2003. № 1. С.39-42.

7. Матвеев В.Ю. Особенности диагностирования топливной аппаратуры дизельных двигателей / В.Ю. Матвеев, А.Е. Крупин, А.В. Щагвин // Агротехника и энергообеспечение. 2016. № 1 (10). C.11-20. 
ВІСНИК

ОДЕСЬКОГО НАЦІОНАЛЬНОГО

МОРСЬКОГО УНІВЕРСИТЕТУ

№ 1 (61), 2020
HERALD

OF THE ODESSA NATIONAL

MARITIME UNIVERSITY

8. Захаров Ю.А. Актуальность проведения диагностики, испытания и проверки форсунок дизельных двигателей мобильных машин / Ю.А. Захаров, Е.А. Кульков // Современные научные исследования и инноващии. 2015. № 3 (2). С.156-160.

9. Астахов И.В. Топливные системы и экономичность дизелей / И.В. Астахов. Л.: Машиностроение, 1990. 288 с.

10. Чванов К.Г. Современные подходы к оценке технического состояния дизельной топливной аппаратуры / К.Г. Чванов // Сетевое научно-практическое издание «Управление рисками в АПК». 2016. № 7. С.13-19.

11. Половинка Э.М. Влияние начальных условий на проиесс топливоподачи среднеоборотного судового дизеля на переменных режимах / Э.М. Половинка, Н.В. Слободянюк // Н. журнал «American Scientific Journal», United States. 2018. № 19. C.51-59.

12. Половинка Э.М. Динамические испытания системы топливоподачи судового среднеоборотного дизеля / Э.М. Половинка, Н.В. Слободянюк // Н. журнал «Молодий вчений». № 5. Херсон, 2019. С.19-24.

13. Небеснов В.И. Об электрическом моделировании режимов совместной работы корпуса судна, винтов и двигателей / В.И. Небеснов, В.А. Плотников // Журнал «Судоремонт и судостроение. Bылn. 2. Одесса, 1963. C.37-44.

14. Беллман Р. Прикладные задачи динамического программирования / Р. Беллман, С. Дрейфус. М.: Наука, 1965. 320 с.

15. Мойсеев Н.Н. Численные методы динамического программирования / Н.Н. Мойсеев. М.: Наука, 1985. 360 c.

\section{REFERANCES}

1. Lepeshkin, D.I. (2013) Experimental studies on the oscillography of processes in diesel fuel equipment, Omsk Scientific Bulletin, 3 (123), 150-153. [in Russian].

2. Smirnov, A.M. (2014) Diagnostic method for diesel fuel high pressure equipment, Omsk Scientific Bulletin, 3 (133), 133-136. [in Russian].

3. Saenko, M.M. (2013) The influence of various factors on the size and uniformity of fuel supply to the diesel cylinders, Bulletin of SibADI, 4 (32), 29-35. [in Russian].

4. Saenko, M.M. (2014) The influence of the design of the devices of the diesel fuel system on the amount of fuel supply, Vestnik SibADI, 6 (40), 49-55. [in Russian]. 
5. Saenko, M.M. (2015) Analysis of existing test methods for devices of diesel fuel systems during maintenance during operation, Bulletin of SibADI, 2 (42), 40-46. [in Russian].

6. Potomarev, M.N. (2003) Transients in high pressure lines of diesel fuel equipment, Bulletin of the ore, ser. Engineering Research, 1, 39-42. [in Russian].

7. Matveev, V.Yu. (2016) Features of diagnosing diesel engine fuel equipment, Agrotechnics and energy supply, 1 (10), 11-20. [in Russian].

8. Zakharov, Yu.A. (2015) The relevance of diagnostics, testing and verification of nozzles of diesel engines of mobile machines, Modern scientific research and innovation, 3 (2), 156-160. [in Russian].

9. Astakhov, I.V. (1990) Fuel systems and diesel efficiency, Engineering, 288. [in Russian].

10. Chvanov, K.G. (2016) Modern approaches to assessing the technical condition of diesel fuel equipment, Network scientific and practical publication "Risk Management in the AIC», 7, 13-19. [in Russian].

11. Half, E.M. (2018) Influence of initial conditions on the fuel supply process of a medium-speed marine diesel engine under variable conditions, N. American Scientific Journal, 19, 51-59. [in English].

12. Half, E.M. (2019) Dynamic tests of the fuel supply system of a marine medium-speed diesel engine, N. Magazine «Molody Vcheniy», 5, 19-24. [in Russian].

13. Nebesnov, V.I. (1963) About electrical modeling of modes of joint operation of the hull, propellers and engines, Journal «Ship repair and shipbuilding», 2, 37-44. [in Russian].

14. Bellman, R. (1965) Applied problems of dynamic programming, Nauka, 320. [in Russian].

15. Moiseev, N.N. (1985) Numerical methods of dynamic programming, Nauka, 360. [in Russian].

Статья надійшла до редакиії 20.02.20

Посилання на статтю: Усов А.В., Половинка Э.М., Слободянюк Н.В. Оптимальное управление судовым дизелем при нестационарных режимах // Вісник Одеського національного морського університету: Зб. наук. праць, 2020. № 1(61). C. 131-139. DOI 10.47049/2226-1893-2020-1-131-139.

Article received 20.02.20

Reference a JournalArtic: Usov, A., Polovinka, E., Slobodiynyk N. Optimal management by ship diesel at non-stationary modes. 1(61), 131-139 // Herald of the Odessa national maritime university. DOI 10.47049/2226-1893-2020-1-131-139. 